\title{
О СВЯЗИ НЕКОТОРЫХ ГРУПП, ПОРОЖДЕННЫХ З-ТРАНСПОЗИЦИЯМИ, С ГРУППАМИ КОКСТЕРА ${ }^{1}$
}

\author{
В. М. Синицин, А. И. Созутов
}

Группы Кокстера, более известные как группы, порожденные отражениями, имеют многочисленные приложения в различных областях математики и за ее пределами. Группы с 3-транспозициями Фишера также связаны со многими структурами: конечные простые группы, тройные графы, геометрии различных пространств, алгебры Ли и др. Пересечение этих классов групп состоит из конечных групп Вейля $W\left(A_{n}\right) \simeq S_{n+1}, W\left(D_{n}\right), W\left(E_{n}\right)(n=6,7,8)$ простых конечномерных алгебр и групп Ли. В работе продолжается исследование связи между конечными группами $S p_{2 l}(2)$ и $O_{2 l}^{ \pm}(2)$ из пп. (ii)-(iii) теоремы Фишера и бесконечными группами Кокстера. Организующей основой исследуемой связи являются общие графыдеревья Кокстера $\Gamma_{n}$ с вершинами $1, \ldots, n$. Каждой вершине $i$ графа $\Gamma_{n}$ ставятся в соответствие порождающая инволюция (отражение) $s_{i}$ группы Кокстера $G_{n}$, базисный вектор $e_{i}$ пространства $V_{n}$ над полем $F_{2}$ из двух элементов и порождающая трансвекция $w_{i}$ подгруппы $W_{n}=\left\langle w_{1}, \ldots, w_{n}\right\rangle$ из $S L\left(V_{n}\right)=S L_{n}(2)$. Графу $\Gamma_{n}$ соответствует точно одна группа Кокстера ранга $n: G_{n}=\left\langle s_{1}, \ldots, s_{n} \mid\left(s_{i} s_{j}\right)^{m_{i j}}, m_{i j} \leq 3\right\rangle$, где $m_{i i}=1,1 \leq i<j \leq n$ и $m_{i j}=3$ или $m_{i j}=2$ в зависимости от того, есть в $\Gamma_{n}$ ребро $(i, j)$ или такого ребра нет. Определенная по графу $\Gamma_{n}$ форма превращает $V_{n}$ в ортогональное пространство, группа изометрий $W_{n}$ которого порождается указанными выше трансвекциями (3-транспозциями) $w_{1}, \ldots, w_{n}$; при этом в $W_{n}$ выполняются соотношения $\left(w_{i} w_{j}\right)^{m_{i j}}=1$, и, значит, отображение $s_{i} \rightarrow w_{i}(i=1, \ldots, n)$ продолжается до сюрьективного гомоморфима $G_{n} \rightarrow W_{n}$. В предыдущей работе авторов для всех групп $W_{n}=O_{2 l}^{ \pm}(2)(n=2 l \geq 6)$ и $W_{n}=S p_{2 l}(2)(n=2 l+1 \geq 7)$ был указан алгоритм перечисления соответствующих им графов-деревьев $\Gamma_{n}$ с помощью группировки их по $E$-сериям вложенных друг в друга графов. В настоящей работе установлена самая тесная генетическая связь между группами $O_{2 l}^{ \pm}(2), S p_{2 l}(2) \times \mathbb{Z}_{2}$ $(3 \leq l \leq 10)$ и соответствующими (бесконечными) группами Кокстера $G_{n}$ с разницей в генетических кодах точно на один ген (соотношение). Для групп $W_{n}$ с графами $\Gamma_{n}$ из $E$-серий $\left\{E_{n}\right\},\left\{I_{n}\right\},\left\{J_{n}\right\}$ и $\left\{K_{n}\right\}$ дополнительные слова-соотношения выписаны в явном виде.

Ключевые слова: группы с 3-транспозициями, графы и группы Кокстера, генетические коды.

V. M. Sinitsin, A. I. Sozutov. On the connection of some groups generated by 3-transpositions with Coxeter groups.

Coxeter groups, more commonly known as reflection-generated groups, have numerous applications in various fields of mathematics and beyond. Groups with Fischer's 3-transpositions are also related to many structures: finite simple groups, triple graphs, geometries of various spaces, Lie algebras, etc. The intersection of these classes of groups consists of finite Weyl groups $W\left(A_{n}\right) \simeq S_{n+1}, W\left(D_{n}\right)$, and $W\left(E_{n}\right)(n=6,7,8)$ of simple finite-dimensional algebras and Lie groups. The paper continues the study of the connection between the finite groups $S p_{2 l}(2)$ and $O_{2 l}^{ \pm}(2)$ from clauses (ii)-(iii) of Fischer's theorem and infinite Coxeter groups. The organizing basis of the connection under study is general Coxeter tree graphs $\Gamma_{n}$ with vertices $1, \ldots, n$. To each vertex $i$ of the graph $\Gamma_{n}$, we assign the generating involution (reflection) $s_{i}$ of the Coxeter group $G_{n}$, the basis vector $e_{i}$ of the space $V_{n}$ over the field $F_{2}$ of two elements, and the generating transvection $w_{i}$ of the subgroup $W_{n}=$ $\left\langle w_{1}, \ldots, w_{n}\right\rangle$ of $S L\left(V_{n}\right)=S L_{n}(2)$. The graph $\Gamma_{n}$ corresponds to exactly one Coxeter group of rank $n: G_{n}=$ $\left\langle s_{1}, \ldots, s_{n} \mid\left(s_{i} s_{j}\right)^{m_{i j}}, m_{i j} \leq 3\right\rangle$, where $m_{i i}=1,1 \leq i<j \leq n$, and $m_{i j}=3$ or $m_{i j}=2$ depending on whether $\Gamma_{n}$ contains the edge $(i, j)$. The form defined by the graph $\Gamma_{n}$ turns $V_{n}$ into an orthogonal space whose isometry group $W_{n}$ is generated by the mentioned transvections (3-transpositions) $w_{1}, \ldots, w_{n}$; in this case, the relations $\left(w_{i} w_{j}\right)^{m_{i j}}=1$ hold in $W_{n}$ and, therefore, the mapping $s_{i} \rightarrow w_{i}(i=1, \ldots, n)$ is continued to the surjective homomorphism $G_{n} \rightarrow W_{n}$. In the authors' previous paper, for all groups $W_{n}=O_{2 l}^{ \pm}(2)(n=2 l \geq 6)$ and $W_{n}=S p_{2 l}(2)(n=2 l+1 \geq 7)$, an algorithm was given for enumerating the corresponding tree graphs $\Gamma_{n}$ by grouping them according to $E$-series of nested graphs. In the present paper, a close genetic connection is established between the groups $O_{2 l}^{ \pm}(2)$ and $S p_{2 l}(2) \times \mathbb{Z}_{2}(3 \leq l \leq 10)$ and the corresponding (infinite) Coxeter groups $G_{n}$ with the difference in their genetic codes by exactly one gene (relation). For the groups $W_{n}$ with the graphs $\Gamma_{n}$ from the $E$-series $\left\{E_{n}\right\},\left\{I_{n}\right\},\left\{J_{n}\right\}$, and $\left\{K_{n}\right\}$, additional word relations are written explicitly.

Keywords: groups with 3-transpositions, Coxeter graphs and groups, genetic codes.

MSC: $20 \mathrm{C} 40$

DOI: $10.21538 / 0134-4889-2020-26-4-234-243$

${ }^{1}$ Исследование выполнено при финансовой поддержке РФФИ в рамках научного проекта № 19-0100566 A. 


\section{Введение}

Множество $D=a^{G}$ инволюций группы $G$ называется классом 3-транспозиций, если $|a b| \leq 3$ для любых $a, b \in D[1 ; 2]$; подгруппы $H=\langle D \cap H\rangle$ из $G$ называются $D$-подгруппами [1]. Если в $G$ нет $D$-подгрупп порядков 18 и 54, то $G$ называется группой с симплектическими 3-транспозиииями [3] (в работе [4] $G$ называлась группой muna $\Sigma_{4}$.) В известной теореме Б. Фишера $[1 ; 2$, теорема 2.58] группами с симплектическими 3-транспозициями являются симметрические группы $S_{n}$, симплектические группы $S p_{2 l}(2)$ и ортогональные группы $O_{2 l}^{ \pm}(2)$. Б. Фишер в [1] использует описание этих групп из [5].

Группы с 3-транспозициями связаны со многими математическими структурами; это конечные простые группы [2;6], тройные графы [2, с.125], геометрии пространств Фишера, геометрии ортогональных, симплектических, унитарных и др. пространств [3;5;7;8], алгебры Ли [9; 10], алгебры вершинных операторов и др. (см., например, [11;12]).

В данной статье установлена тесная "генетическая связь" групп $S p_{2 l}(2)$ и $O_{2 l}^{ \pm}(2)$ с некоторыми группами (системами) Кокстера, известными во многих областях математики как группы, порожденные отражениями [13, с. 286-293; 14, гл. 9]. Генетическим кодом или просто кодом группы $G$ называется перечень ее порождающих элементов $S$ и определяющих соотношений $R$ (см. [14, с. 10]). Группа Кокстера $G$ (система Кокстера $(G, S)$ ) задается кодом, однозначно определяемым матрицей и графом Кокстера [13, с. 24-25].

В работе доказано, что конечные группы

$$
O_{2 l}^{ \pm}(2)(5 \leq l \leq 10) \text { и } \quad S p_{2 l}(2) \times \mathbb{Z}_{2} \quad(4 \leq l \leq 9)
$$

(см. пояснения в замечании 3) могут быть получены из подходящих бесконечных групп Кокстера

$$
G_{n}=\left\langle s_{1}, \ldots, s_{n} \mid R_{n}\right\rangle \quad(n=2 l \text { и } n=2 l+1 \text { соответственно })
$$

с помощью точно одного дополнительного соотношения.

Системы порождающих 3-транспозиций групп $O_{2 l}^{ \pm}(2)$ и $S p_{2 l}(2)$ с графами-деревьями Кокстера $\Gamma_{n}$, в которых проявляется указанная связь с группами Кокстера $G_{n}$, были частично описаны в [8], а в общем случае - в предыдущей работе авторов (Тр. Ин-та математики и механики УрО РАН. 2016. Т. 22, № 3. С. 251-258). Там же графы $\Gamma_{n}$ были снабжены разметкой, указывающей, для каких групп $W_{n} \leq S L_{n}(2)$ граф $\Gamma_{n}$ является графом Кокстера (подробнее см. предложение 1). Мы рассматриваем четыре серии графов $\Gamma_{n}$ и групп $W_{n}$, детально исследованных в [8]:

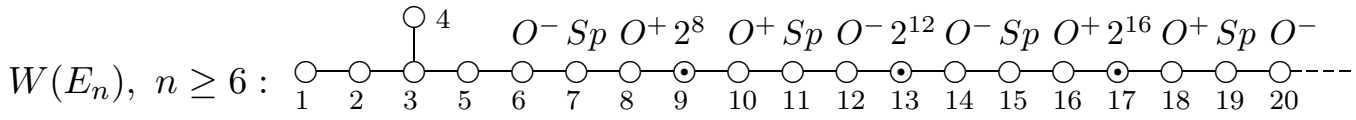

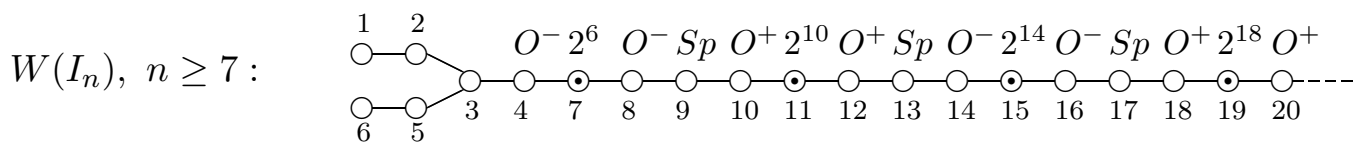

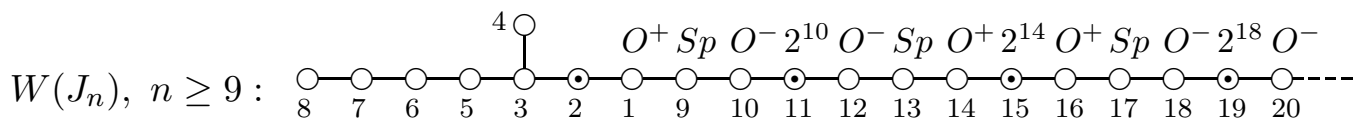

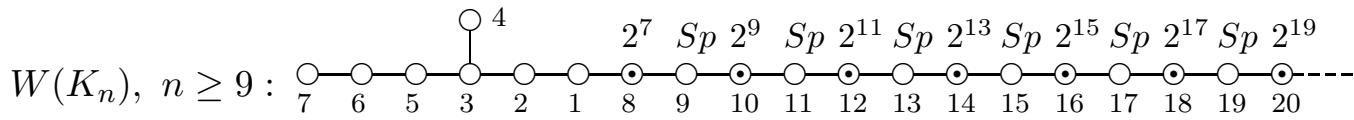

Смысл разметки поясняется в предложении 1. 
Каждому графу-дереву $\Gamma_{n}$ соответствует группа Кокстера $G_{n}=G\left(\Gamma_{n}\right)$ :

$G_{n}=\left\langle s_{1}, \ldots, s_{n}\right| s_{i}^{2},\left(s_{k} s_{j}\right)^{2},\left(s_{i} s_{j}\right)^{3}$, где $\left.1 \leq i, j, k \leq n,(k, j) \notin E\left(\Gamma_{n}\right),(i, j) \in E\left(\Gamma_{n}\right)\right\rangle$.

Через $X_{n}=X_{n}\left(\Gamma_{n}\right)$ обозначим группу

$$
X_{n}=\left\langle s_{1}, \ldots, s_{n} \mid R_{n}, w^{2}\right\rangle,
$$

где $R_{n}$ - соотношения Кокстера из (1), а слово $w=w\left(\Gamma_{n}\right)$ определено в $(3)-(6)$ :

$$
\begin{gathered}
\Gamma_{n}=E_{n}: w=s_{4}^{v} s_{9}, v=s_{3} s_{2} s_{1} s_{5} s_{6} s_{7} s_{8} s_{3} s_{2} s_{5} s_{3} s_{4} s_{6} s_{5} s_{3} s_{2} s_{7} s_{6} s_{5} s_{3} s_{4} s_{1} s_{2} s_{3} s_{5} s_{6} s_{7} s_{8} ; \\
\Gamma_{n}=I_{n}: w=s_{4}^{v} s_{7}, v=s_{3} s_{2} s_{1} s_{5} s_{6} s_{3} s_{2} s_{5} s_{3} s_{4} ; \\
\Gamma_{n}=J_{n}: w=s_{4}^{v} s_{9}, \text { где } v=s_{3} s_{2} s_{1} s_{5} s_{6} s_{7} s_{3} s_{2} s_{5} s_{6} s_{3} s_{5} s_{4} s_{3} s_{2} s_{1} \quad \text { или } \\
v=s_{3} s_{2} s_{1} s_{5} s_{6} s_{7} s_{8} s_{3} s_{2} s_{5} s_{3} s_{4} s_{6} s_{5} s_{3} s_{2} s_{7} s_{6} s_{5} s_{3} s_{4} s_{1} s_{2} s_{3} s_{5} s_{6} s_{7} s_{8} ; \\
\Gamma_{n}=K_{n}: w=s_{4}^{v} s_{8}, \quad v=s_{3} s_{2} s_{1} s_{5} s_{6} s_{7} s_{3} s_{5} s_{6} s_{2} s_{3} s_{5} s_{4} s_{3} s_{2} s_{1} .
\end{gathered}
$$

Сформулируем основной результат данной работы.

Теорема. При $7 \leq n \leq 20$ группы $X_{n}$, заданные копредставлениями (2) и словами $w$ из (3)-(6), конечны, и для них справедливы следующие утверждения.

1. Для групп $X_{n}=X\left(E_{n}\right)$, определенных в (2) и (3), имеют место изоморфизмы

i) $X_{4 k} \simeq O_{4 k}^{-}(2)$ при нечетном $k$ и $X_{4 k} \simeq O_{4 k}^{+}(2)$ при четном $k$;

ii) $X_{4 k+2} \simeq O_{4 k+2}^{-}(2)$ при нечетном $k$ и $X_{4 k+2} \simeq O_{4 k+2}^{+}(2)$ при четном $k$;

iii) $X_{4 k+3} \simeq S p_{4 k+2}(2) \times \mathbb{Z}_{2}$.

2. Для групп $X_{n}=X\left(I_{n}\right)$, определенных в (2) и (4), имеют место изоморфизмы

i) $X_{4 k} \simeq O_{4 k}^{-}(2)$ при четном $k$ и $X_{4 k} \simeq O_{4 k}^{+}(2)$ при нечетном $k$;

ii) $X_{4 k+1} \simeq S p_{4 k}(2) \times \mathbb{Z}_{2}$

iii) $X_{4 k+2} \simeq O_{4 k+2}^{-}(2)$ при нечетном $k$ и $X_{4 k+2} \simeq O_{4 k+2}^{+}(2)$ при четном $k$.

3. Для групп $X_{n}=X\left(J_{n}\right)$, определенных в (2) и (5), имеют место изоморфизмы

i) $X_{4 k} \simeq O_{4 k}^{-}(2)$ при нечетном $k$ и $X_{4 k} \simeq O_{4 k}^{+}(2)$ при четном $k$;

ii) $X_{4 k+1} \simeq S p_{4 k}(2) \times \mathbb{Z}_{2}$;

iii) $X_{4 k+2} \simeq O_{4 k+2}^{+}(2)$ при нечетном $k$ и $X_{4 k+2} \simeq O_{4 k+2}^{-}(2)$ при четном $k$.

4. Группа $X_{2 k+1}=X\left(K_{2 k+1}\right)$, определенная в (2) и (6), изоморфна группе $S p_{2 k}(2) \times \mathbb{Z}_{2}$.

3 а м е ч а н и е 1 . Для групп $O_{2 l}^{ \pm}(2), S p_{2 l}(2) \times \mathbb{Z}_{2}$ из теоремы число найденных кодов различно. Так, например, группы $O_{8}^{-}(2)$ и $O_{12}^{+}(2)$ получили по одному коду, $O_{14}^{ \pm}(2)-$ по два кода (графы $J_{n}$ с “двойной кодировкой”), $O_{16}^{+}(2)-$ три кода, а $S p_{8}(2) \times \mathbb{Z}_{2}$ - четыре кода. 


\section{1. Предварительные результаты}

Поясним подробнее смысл меток над вершинами рассматриваемых графов $\Gamma_{n}$.

Предложение 1. Справедливы следующие утверждения.

1. $\Gamma_{n}$ - граф Кокстера группы $W_{n}=W\left(\Gamma_{n}\right)$ изометрий квадратичной формы $F$ пространства $V_{n}$ над полем $F_{2}$ порлдка 2 с базисом $\left(e_{1}, \ldots, e_{n}\right)$.

2. Если метка вершины $n$ равна $O^{ \pm}$, то $W_{n} \simeq O_{n}^{ \pm}(2)$, при этом $n-$ четное число.

3. Если над верииной $n$ стоит метка $S p$, то $W_{n} \simeq S p_{n-1}(2)$ и $n$ - нечетное число.

4. Если метка вершины $n$ равна $2^{n-1}$, то группа $W_{n}$ обладает нормальной элементарной абелевой 2-подгруппой порядка $2^{n-1}, n-$ нечетное число, $W_{n} \simeq 2^{n-1} . O_{n-1}^{ \pm}(2)$ и метки вериин $n-1$ и $n+1$ совпадают.

5. Последовательность меток $O^{ \pm}$и $S p$ в Е-сериях $\left\{E_{n}\right\},\left\{I_{n}\right\},\left\{J_{n}\right\}$ имеет период 8, m. е. группы $W_{n} u W_{n+8}$ одного типа, а в серии $\left\{K_{n}\right\}$ - период 2.

Д о к а з а т е л ь с т в о. Докажем сначала утверждение 1 . По лемме 20 из [8] $W_{n}=$ $\left\langle w_{1}, \ldots, w_{n}\right\rangle$ - группа изометрий квадратичной формы $F_{n}$ линейного пространства $V_{n}$ над полем $F_{2}$ с базисом $\left(e_{1}, \ldots, e_{n}\right)$, где

$$
F_{n}(x)=\sum_{i \in \Gamma_{n}} x_{i}^{2}+\sum_{(i, j) \in \Gamma_{n}} x_{i} x_{j} \text { для произвольного вектора } x=\sum_{i=1}^{n} x_{i} e_{i} \text { из } V_{n} .
$$

Стандартно определяется симплектическая форма $f_{n}$ на $V_{n}$ :

$$
f_{n}(x, y)=F_{n}(x+y)+F_{n}(x)+F_{n}(y) .
$$

Трансвекции $w_{i}: x \rightarrow x+f_{n}\left(e_{i}, x\right) \cdot e_{i}$, где $i=1, \ldots, n$, являются 3-транспозициями группы $W_{n}$ $[8$, лемма 20], при этом выполняются соотношения

$$
w_{i}^{2}=\left(w_{k} w_{j}\right)^{2}=\left(w_{i} w_{j}\right)^{3}=1, \quad \text { где } 1 \leq i, j, k \leq n, \quad(k, j) \notin E\left(\Gamma_{n}\right), \quad(i, j) \in E\left(\Gamma_{n}\right) .
$$

Следовательно, $\Gamma_{n}$ - граф Кокстера группы $W_{n}$ в системе порождающих 3 -транспозиций $\left\{w_{1}, \ldots, w_{n}\right\}$, и утверждение 1 предложения доказано.

Доказательства утверждений 2-4 дословно повторяют доказательство соответствующей части предложения 1 из работы первого автора (Тр. Ин-та математики и механики УрО PAН. 2019. Т. 25, № 4. С. 184-188). Утверждение 5 для рассматриваемых в работе графов допускает непосредственную проверку.

Предложение доказано.

Нам понадобятся некоторые свойства групп $O_{2 l}^{ \pm}(2)$ и $S p_{2 l}(2)$ из [15, теоремы 6.1.1, 6.3.4, табл. 6.4.1] и [16, пп. 3.1.5., 3.2.1, 3.4.1]. Приведем их в виде предложений.

Предложение 2. При $l \geq 4$ группа $O_{2 l}^{ \pm}(2)$ порождена трансвекииями, сохраняющими соответствуюшую квадратичную форму, ее коммутант $\Omega_{2 l}^{ \pm}(2)$ имеет индекс 2 и является простой группой Шевалле: $\Omega_{2 l}^{+}(2)=D_{l}(2), \Omega_{2 l}^{-}(2)={ }^{2} D_{l}(2)$.

Предложение 3. Группа $S p_{2 l}(2)$ порождена 3-транспозиииями (симплектическими трансвекииями), при $l \geq 3$ проста (совпадает с группой $P S p_{2 l}(2)$ ), не имеет внешних автоморфизмов и является группой Шевалле $C_{l}(2)$.

Обозначим через $x_{1}, \ldots, x_{n}$ образы порождающих элементов $s_{1}, \ldots, s_{n}$ в копредставлениях групп $X_{n}$ из (2). 
Лемма 1. Отображения $s_{1} \rightarrow x_{i}, x_{i} \rightarrow w_{i} u s_{i} \rightarrow w_{i}$ продолжаются до сюрвективных гомоморфизмов $\varphi_{1}: G_{n} \rightarrow X_{n}, \varphi_{2}: X_{n} \rightarrow W_{n} u \varphi_{0}: G_{n} \rightarrow W_{n}$ соответственно так, что $\varphi_{0}=\varphi_{2} \varphi_{1}$ и соответствуюшая диаграмма коммутативна.

Д о к а з а т е л ь с т в о. В силу теоремы Дика [15, теорема 12.2.1], заданий групп $G_{n}(1)$, $X_{n}(2)$ и выполнимости в $W_{n}$ соотношений (9) отображения $s_{1} \rightarrow x_{i}$ и $s_{i} \rightarrow w_{i}$ продолжаются до сюрьективных гомоморфизмов $\varphi_{1}$ и $\varphi_{0}$ соответственно. Для обоснования гомоморфизма $\varphi_{2}$ нужно показать, что $\varphi_{0}\left(w^{2}\right)=1$ в группе $W_{n}$ для соответствующего слова $w$ из $(3)-(6)$.

Рассмотрим случай $\Gamma_{n}=E_{n}$, для которого $w=s_{4}^{v} s_{9}$ из (3). Группа $G_{8}=G\left(E_{8}\right)$ изоморфна группе Вейля $W\left(E_{8}\right)[13]$ и содержится во всех группах $G_{n}=G\left(E_{n}\right)$ при $n \geq 8$. Инволюция $s=s_{4}^{v}$ в группе $W\left(E_{8}\right)$ является симметрией $w_{r}$, определенной максимальным положительным корнем $r=2 p_{1}+4 p_{2}+6 p_{3}+3 p_{4}+5 p_{5}+4 p_{6}+3 p_{7}+2 p_{8}$ в корневой системе типа $E_{8}$ с фундаментальной системой корней $\left\{p_{1}, \ldots, p_{8}\right\}$ (см. [13, с. 314]). Ввиду $\left[8\right.$, лемма 3] $\varphi_{0}\left(s_{r}\right)=w_{r^{\prime}}$, где $r^{\prime}=e_{4}+e_{5}+e_{7} \in V_{n}$. В пространстве $V_{n}$ векторы $r^{\prime}$ и $e_{9}$ ортогональны относительно формы $f_{n}$, и потому $\varphi_{0}\left(w^{2}\right)=\left(w_{r^{\prime}} w_{9}\right)^{2}=1$.

Далее, серия графов $I_{n}$ начинается с графа $E_{6}$, а слово $w=s_{4}^{v} s_{7}$ берется из соотношения (4). Как и выше, $W\left(E_{6}\right) \leq G_{n}=G\left(I_{n}\right)$ при $n \geq 6$, и инволюция $s_{4}^{v}$ является симметрией $w_{r}$, определенной максимальным положительным корнем $r=p_{1}+2 p_{2}+3 p_{3}+2 p_{4}+2 p_{5}+p_{6}$ корневой системы типа $E_{6}[13$, с. 310$]$. В пространстве $V\left(I_{7}\right)$ векторы $r^{\prime}=e_{1}+e_{3}+e_{6}$ и $e_{7}$ ортогональны относительно формы $f_{n}$, и $\varphi_{0}\left(w^{2}\right)=\left(w_{r^{\prime}} w_{7}\right)^{2}=1$.

Для групп $W_{n}=W\left(J_{n}\right)$ в (5) дано два значения слова $w$. Как и в случае $\Gamma_{n}=E_{n}$, используем вложения $W\left(E_{8}\right) \leq G_{n}$ при $n \geq 9$. Для второго значения слова $w$ доказательство равенства $\varphi_{0}\left(w^{2}\right)=1$ дословно повторяет доказательство для случая $\Gamma_{n}=E_{n}$. При первом значении слова $w$ инволюция $s_{4}^{v}$ является симметрией $w_{r}$, определенной максимальным положительным корнем $r=2 p_{1}+3 p_{2}+4 p_{3}+2 p_{4}+3 p_{5}+2 p_{6}+p_{7}$ корневой системы типа $E_{7}$ (см. [13, с. 312]). Для "проекции" $r^{\prime}=e_{2}+e_{5}+e_{7}$ в $V\left(E_{9}\right)$ выполняются равенства $f_{9}\left(r^{\prime}, e_{9}\right)=0$ и $\varphi_{0}\left(w^{2}\right)=\left(w_{r^{\prime}} w_{9}\right)^{2}=1$.

Наконец, для $\Gamma_{n}=K_{n}$ аналогично получаем $W\left(E_{7}\right) \leq G_{n}, s_{4}^{v}=w_{r}-$ симметрия из $W\left(E_{7}\right)$ для $r=2 p_{1}+3 p_{2}+4 p_{3}+2 p_{4}+3 p_{5}+2 p_{6}+p_{7}, r^{\prime}=e_{2}+e_{5}+e_{7}, f_{8}\left(w_{r^{\prime}}, e_{8}\right)=0$ и $\varphi_{0}\left(w^{2}\right)=\left(w_{r^{\prime}} w_{8}\right)^{2}=1$.

Лемма доказана.

3 а м е ч а н и е 2. Используя геометрическое представление групп Кокстера [13, §4], можно показать, что порядки элементов $w\left(s_{1}, \ldots, s_{m}\right)$ из $(3)-(6)$ в группах $G_{m}$ бесконечны.

Согласно заданию $(2)$ групп $X_{n}$ (генетический) код группы $X_{n}$ содержится в коде группы $X_{n+1}$. Следовательно, группа $X_{n}$ вкладывается в $X_{n+1}$ либо изоморфно, либо как некоторая фактор-группа $\bar{X}_{n}$ группы $X_{n}$. С помощью системы GAP для $n \leq 20$ были найдены индексы $\left[X_{n+1}: X_{n}\right]$ и порядки групп $X_{n}$, они оказались конечными. Результат сформулируем в виде утверждения.

Предложение 4. В предположении изоморфной вложимости $X_{n}<X_{n+1} \partial \Omega я 6 \leq n \leq 20$ порядки групп $X_{n}=X\left(E_{n}\right)$ следующие:

$\left|X\left(E_{6}\right)\right|=51840=\left|W\left(E_{6}\right)\right|=\left|O_{6}^{-}(2)\right| ;$

$\left|X\left(E_{7}\right)\right|=2903040=\left|W\left(E_{7}\right)\right|=2 \cdot\left|S p_{6}(2)\right| ;$

$\left|X\left(E_{8}\right)\right|=240 \cdot\left|W\left(E_{7}\right)\right|=\left|W\left(E_{8}\right)\right|=2 \cdot\left|O_{8}^{+}(2)\right|$;

$\left|X\left(E_{9}\right)\right|=256 \cdot\left|W\left(E_{8}\right)\right|=2^{9} \cdot\left|O_{8}^{+}(2)\right|$;

$\left|X\left(E_{10}\right)\right|=527 \cdot\left|X\left(E_{9}\right)\right|=2 \cdot\left|O_{10}^{+}(2)\right|$;

$\left|X\left(E_{11}\right)\right|=1056 \cdot\left|X\left(E_{10}\right)\right|=2^{2} \cdot\left|S p_{10}(2)\right|$;

$\left|X\left(E_{12}\right)\right|=2080 \cdot\left|X\left(E_{11}\right)\right|=2 \cdot\left|O_{12}^{-}(2)\right|$;

$\left|X\left(E_{13}\right)\right|=4096 \cdot\left|X\left(E_{12}\right)\right|=2^{13} \cdot\left|O_{12}^{-}(2)\right|$;

$\left|X\left(E_{14}\right)\right|=8127 \cdot\left|X\left(E_{13}\right)\right|=2 \cdot\left|O_{14}^{-}(2)\right|$;

$\left|X\left(E_{15}\right)\right|=16256 \cdot\left|X\left(E_{14}\right)\right|=2^{2} \cdot\left|S p_{14}(2)\right|$;

$\left|X\left(E_{16}\right)\right|=32640 \cdot\left|X\left(E_{15}\right)\right|=2 \cdot\left|O_{16}^{+}(2)\right|$; 
$\left|X\left(E_{17}\right)\right|=65536 \cdot\left|X\left(E_{16}\right)\right|=2^{17} \cdot\left|O_{16}^{+}(2)\right| ;$

$\left|X\left(E_{18}\right)\right|=131327 \cdot\left|X\left(E_{17}\right)\right|=2 \cdot\left|O_{18}^{+}(2)\right| ;$

$\left|X\left(E_{19}\right)\right|=262656 \cdot\left|X\left(E_{18}\right)\right|=2^{2} \cdot\left|S p_{18}(2)\right| ;$

$\left|X\left(E_{20}\right)\right|=524800 \cdot\left|X\left(E_{19}\right)\right|=2 \cdot\left|O_{20}^{-}(2)\right|$.

В силу леммы 1 порядок группы $X_{n}$ делится на порядок группы $W_{n}$ (ее тип определяется по предложению 1). Поэтому если предположение $X_{n}<X_{n+1}$ нарушается при некотором $n=m$, для всех $n \geq m$ порядки $X_{n}$ будут точно в два раза меньше порядков, приведенных в предложении 4. Отметим, что равенства $\left|X_{4 k+3}\right|=\left|S p_{4 k+2}\right|$ невозможны, поскольку иначе $\left|X_{4 k+4}\right|=\frac{1}{2}\left|O_{4 k+4}^{-}(2)\right|$ - при четном $k$ и $\left|X_{4 k+4}\right|=\frac{1}{2}\left|O_{4 k+4}^{+}(2)\right|-$ при нечетном $k$, что противоречит лемме 1. Итак, порядки интересующих нас групп либо перечислены в предложении 4 , либо в два раза меньше порядков, в нем приведенных. Та же ситуация возникает для групп $X\left(J_{n}\right)$ и $X\left(K_{n}\right)$. Для групп $X\left(I_{n}\right)$, рассмотренных в работе первого автора 2019 г., отмеченной выше, такой дихотомии не возникало.

Результаты проведенных расчетов на компьютере сформулируем в следующем виде.

Предложение 5. Пусть $X_{n} \in\left\{X\left(E_{n}\right), X\left(J_{n}\right)\right\}$. Тогда $\left|X_{n}\right| \in\left\{2\left|W_{n}\right|, 4\left|W_{n}\right|\right\}$ при $W_{n} \simeq$ $S p_{n-1}(2)$ u $\left|X_{n}\right| \in\left\{\left|W_{n}\right|, 2\left|W_{n}\right|\right\}$ при $W_{n} \simeq O_{n}^{ \pm}(2)$. Eсли $X_{n}=X\left(K_{n}\right)$ nри $n=2 l+1$, то $W_{n} \simeq S p_{2 l}(2) u\left|X_{n}\right| \in\left\{\left|W_{n}\right|, 2\left|W_{n}\right|\right\}$.

Заметим, что во всех случаях $\left|X_{20}\right|>2 \cdot 10^{57}$, а порядок спорадической группы $F_{1}$ ("монстра" или "дружественного гиганта") примерно равен $8 \cdot 10^{53}[15$, с. 6$]$.

\section{2. Доказательство теоремы}

Обозначим через $H_{n}$ коммутант группы $G_{n}$. Как следует из $[14$, с. 180$],\left[G_{n}: H_{n}\right]=2$ и $G_{n}=H_{n} \lambda\left\langle s_{1}\right\rangle$. В леммах $2-5$, предусматривая возможность других заданий групп $X_{n}$, будем предполагать, что $X_{n}=\left\langle s_{1}, \ldots, s_{n} \mid R_{n} \cup T_{n}\right\rangle$, где $\left\langle s_{1}, \ldots, s_{n} \mid R_{n}\right\rangle=G_{n}, T_{n} \subseteq H_{n}$, и для всех слов $w\left(s_{1}, \ldots, s_{m}\right) \in T_{n}$ их значения $w\left(w_{1}, \ldots, w_{m}\right)$ в группах $W_{n}$ равны 1 (по лемме 1 ).

Лемма 2. Коммутант $Y_{n}$ группь $X_{n}$ порожден элементами $x_{i} x_{j}$, где $(i, j) \in E\left(\Gamma_{n}\right)$, состоит из всех элементов группь $X_{n}$ четной длины в алфавите $\left\{x_{1}, \ldots, x_{n}\right\},\left[X_{n}: Y_{n}\right]=2$ и $X_{n}=Y_{n} \lambda\left\langle x_{1}\right\rangle$. Ограничение гомоморфизма $\varphi_{1}: G_{n} \rightarrow X_{n}$ на $H_{n}$ совпадает с сюрвективным гомоморфизмом $\varphi: H_{n} \rightarrow Y_{n}$.

Д о к а з а т е л ь с т в о. Повторим рассуждения из [14, с. 180] для групп $Y_{n}$. При $(i, j) \in$ $E\left(\Gamma_{n}\right)$ имеем $\left(x_{i} x_{j}\right)^{3}=1$ и $x_{i} x_{j}=x_{j} x_{i} x_{j} x_{i}=\left[x_{j}, x_{i}\right] \in Y_{n}$. Граф $\Gamma_{n}$ связен, и для любых его различных вершин $i, k$ существует соединяющий их путь $i=i_{1}, i_{2}, \ldots, i_{m}=k$. Отсюда $x_{i} x_{k}=x_{i} x_{i_{2}} \cdot x_{i_{2}} x_{i_{3}} \cdot \ldots \cdot x_{i_{m-1}} x_{i_{m}}$, поэтому $x_{i} x_{k} \in Y_{n}$ и $Y_{n}$ содержит все элементы четной длины из $X_{n}$. Определяющие соотношения группы Кокстера $G_{n}$ и дополнительные соотношения из $T_{n}$ как элементы подгруппы $H_{n}$ имеют четную длину в алфавите $S_{n}$. Поэтому каждый элемент группы $X_{n}$ либо четен, либо нечетен, и элементы четной длины составляют в $X_{n}$ подгруппу $Y_{n}$ индекса 2 и, очевидно, $X_{n}=Y_{n} \lambda\left\langle x_{1}\right\rangle$. Наконец, сюрьективность гомоморфизма $\varphi: H_{n} \rightarrow Y_{n}$ следует из включения $\operatorname{Ker} \varphi_{1} \leq H_{n}$.

Лемма доказана.

3 а м е ч а н и е 3 . По теореме Фишера класс 3 -транспозиций $D$ группы $S p_{2 l}(2)$ совпадает с множеством ее симплектических трансвекций [2, теорема 2.58]. В прямом произведении $G=$ $S p_{2 l}(2) \times \mathbb{Z}_{2}$, где $\mathbb{Z}_{2}=\langle z\rangle$ - группа порядка 2 , множество $D z$ является классом сопряженных 3 -транспозиций, поскольку $|a z \cdot b z|=|a b|$ для любых $a, b \in D$. Далее, группа $S p_{2 l}(2)$ проста (предложение 2), и ввиду леммы $2 a_{1} \cdot \ldots \cdot a_{2 k+1}=1$ для некоторых $a_{1}, \ldots, a_{2 k+1} \in D$ и $a_{1} z \cdot \ldots \cdot a_{2 k+1} z=z$. Следовательно, $G=\langle D z\rangle=S p_{2 l}(2) \times \mathbb{Z}_{2}-$ группа, порожденная классом сопряженных 3 -транспозиций. 
Лемма 3. Если $n=2 l+1 \geq 9, W_{n} \simeq S p_{2 l}(2) u\left|X_{n}\right|=2\left|S p_{2 l}(2)\right|$, mo $X_{n} \simeq S p_{2 l}(2) \times \mathbb{Z}_{2}$. Если $n=2 l \geq 6$ u $\left|X_{n}\right|=\left|O_{2 l}^{ \pm}(2)\right|$, mо $X_{n} \simeq O_{2 l}^{ \pm}(2)$.

Д о к а з а т е л ь с т в о. По лемме 1 существует сюрьективный гомоморфизм

$$
\varphi_{2}: X_{2 l+1} \rightarrow S p_{2 l}(2),
$$

и ввиду предложения $3 \operatorname{Ker} \varphi_{2}=Z\left(X_{n}\right)$. С другой стороны, по лемме $2\left[X_{n}: Y_{n}\right]=2$, что ввиду предложения 3 влечет изоморфизмы $Y_{n} \simeq S p_{2 l}(2)$ и $X_{n}=Y_{n} \times \mathbb{Z}_{2} \simeq S p_{2 l}(2) \times \mathbb{Z}_{2}$. Второе утверждение очевидно.

Лемма доказана.

Лемма 4. Eсли $n=2 l+1 \geq 9 u W_{n} \simeq S p_{2 l}(2), m o\left|X_{n}\right|=2\left|S p_{2 l}(2)\right|$.

Д о к а з а т е л ь с т в о. Предположим, что $\left|X_{n}\right| \neq 2\left|S p_{2 l}(2)\right|$. Тогда по предложению 5 $\left|X_{n}\right|=4\left|S p_{2 l}(2)\right|$. Пусть $\varphi_{2}: X_{n} \rightarrow S p_{2 l}(2)$ - сюрьективный гомоморфизм из леммы 1 . Так как группа $S p_{2 l}(2)$ проста (предложение 3$)$ и $\left[X_{n}: Y_{n}\right]=2$ (лемма 2), то $\varphi_{2}\left(Y_{n}\right)=\varphi_{2}\left(X_{n}\right)$. Обозначим через $Z$ ядро индуцированного $\varphi_{2}$ гомоморфизма $Y_{n} \rightarrow S p_{2 l}(2)$. Тогда $|Z|=2$ и, следовательно, $Z=Z\left(Y_{n}\right)$. По лемме $2 Y_{n}^{\prime}=Y_{n}$, значит, $Y_{n}$ - нерасщепляемое расширение группы порядка 2 при помощи группы $S p_{2 l}(2)$. Поэтому ввиду [2, с. 53] 2 делит порядок мультипликатора Шура группы $S p_{2 l}(2)$. Однако мультипликатор Шура группы $S p_{2 l}(2)$ при $l>3$ тривиален $[2$, табл. $4.1 ; 17]$. Полученное противоречие доказывает лемму.

Лемма 5. Если $n=2 l \geq 10 u W_{n} \simeq O_{2 l}^{ \pm}(2)$, mo $X_{n} \simeq O_{2 l}^{ \pm}(2) u Y_{n} \simeq \Omega_{2 l}^{ \pm}(2)$.

Д о к а з а т е л ь с т в о. В силу предложения 5 либо $\left|X_{n}\right|=\left|O_{2 l}^{ \pm}(2)\right|$, либо $\left|X_{n}\right|=$ $2\left|O_{2 l}^{ \pm}(2)\right|$. Допустим, что $\left|X_{n}\right|=2\left|O_{2 l}^{ \pm}(2)\right|$. Пусть $\varphi_{2}: X_{n} \rightarrow O_{2 l}^{ \pm}(2)$ - сюрьективный гомоморфизм из леммы 1 . В силу леммы $2 Y_{n}=X_{n}^{\prime}$ и $\left[X_{n}: Y_{n}\right]=2$. Поэтому ввиду предложения 2 $\varphi_{2}\left(Y_{n}\right)=W_{n}^{\prime} \simeq \Omega_{2 l}^{ \pm}(2)$ и, следовательно, $Y_{n}$ - нерасщепляемое расширение группы порядка 2 при помощи группы $O_{2 l}^{ \pm}(2)$. Поэтому ввиду [2, с. 53] 2 делит порядок мультипликатора Шура группы $O_{2 l}^{ \pm}(2)$. Однако мультипликатор Шура группы $O_{2 l}^{ \pm}(2)$ при $l>4$ тривиален [2, табл. 4.1; 17]. Полученное противоречие доказывает лемму.

Д о к а з а т е л ь с т в о теоремы. Заметим, что в силу лемм 4,5 все группы $X_{n}$, определенные в соотношениях (2)-(6), удовлетворяют условиям леммы 3 . Для групп $X_{n}=X\left(K_{n}\right)$ теорема следует из предложений 1,5 и леммы 3 , а для групп $X_{n}=X\left(I_{n}\right)$ утверждение теоремы доказано в работе первого автора 2019 г., отмеченной выше.

Для групп $X_{n}$ с графами $\Gamma_{n}=E_{n}$ теорема следует из лемм 3,5 и предложения 1 о разметке графов $E_{n}$. Согласно лемме 3 и разметке (предложение 1 )

$$
X_{4 k+3} \simeq S p_{4 k+2}(2) \times \mathbb{Z}_{2}, \quad Y_{4 k+3} \simeq S p_{4 k+2}(2) \quad \text { и } \quad X_{4 k+\delta} \simeq O_{4 k+\delta}^{ \pm}(2), \quad Y_{4 k+\delta} \simeq \Omega_{4 k+\delta}^{ \pm}(2),
$$

где $\delta=0,2$. Знак \pm определяется по разметке графов $E_{n}$.

Аналогично, группы $X_{n}=X\left(J_{n}\right)$ удовлетворяют условиям леммы 3 , по которой $Y_{n} \simeq$ $S p_{2 l}(2)$ и $X_{n} \simeq S p_{2 l}(2) \times \mathbb{Z}_{2}$ при $W_{n} \simeq S p_{2 l}(2)$ и $X_{n} \simeq O_{2 l}^{ \pm}(2)$ и $Y_{n} \simeq \Omega_{2 l}^{ \pm}(2)$ при $n=2 l$. Согласно разметке графа $J_{n}$ имеем $X_{4 k+1} \simeq S p_{4 k}(2) \times Z_{2}$ и $X_{n} \simeq O_{2 l}^{ \pm}(2)$ для $n=2 l$. Знак \pm , как и выше, определяется по разметке графов $J_{n}$.

Теорема доказана.

Подведем итоги. Для групп $W_{n} \in\left\{S p_{2 l}(2), O_{2 l}^{ \pm}(2)\right\}$ указаны системы $\left\{w_{1}, \ldots, w_{n}\right\}$ порождающих их симплектических трансвекций (3-транспозиций), порядки попарных произведений которых заданы графами-деревьями Кокстера $\Gamma_{n}$ :

$$
w_{i}^{2}=1,\left(w_{k} w_{j}\right)^{2}=1,\left(w_{i} w_{j}\right)^{3}=1, \text { где } 1 \leq i, j, k \leq n,(k, j) \notin E\left(\Gamma_{n}\right), \quad(i, j) \in E\left(\Gamma_{n}\right) .
$$


Каждый граф $\Gamma_{n}$ однозначно определяет группу Кокстера $G_{n}$ :

$$
\left.G_{n}=\left\langle s_{1}, \ldots, s_{n}\right| s_{i}^{2}=\left(s_{k} s_{j}\right)^{2}=\left(s_{i} s_{j}\right)^{3}=1, \text { где } 1 \leq i, j, k \leq n, \quad(k, j) \notin E\left(\Gamma_{n}\right), \quad(i, j) \in E\left(\Gamma_{n}\right)\right\rangle .
$$

По теореме Дика группа $W_{n}$ изоморфна фактор-группе группы $G_{n}$ (в этом нет ничего особенного, поскольку каждая порожденная инволюциями группа изоморфна фактор-группу подходящей группы Кокстера). Особенность заключается в "близком генетическом родстве" групп $W_{n}$ и $G_{n}$, несмотря на рост рангов: генетические коды групn $\left.O_{2 l}^{ \pm}(2)\right\}(n=2 l)$ u групn $S p_{2 l}(2) \times \mathbb{Z}_{2}(n=2 l+1)$ состоят из кодов групп $G_{n}$ и точно одного дополнительного генасоотношения $w^{2}$. При этом $w$ есть произведение двух инволюций, одна из которых $-s_{m+1}$ $(6 \leq m \leq 8)-$ принадлежит порождающему множеству, а вторая является симметрией $w_{r}$, определенной максимальным положительным корнем корневой системы типа $E_{m}$ с конечной группой Вейля $G_{m}=W\left(E_{m}\right)$. Сериям графов $E_{n}, I_{n}, J_{n}$ соответствуют цепи $G_{m}<G_{m+1}<$ $\ldots<G_{n}<\ldots$ вложенных друг в друга групп Кокстера. В работе для $n \leq 20$ подтверждена гипотеза о превращении такой цепи в цепь $W_{m}<W_{m+1}<\ldots<W_{n}<\ldots$ конечных групп $W_{n}$ с 3-транспозициями всего одним дополнительным словом-соотношением $w^{2}$, принадлежащим группе $G_{m+1}$. Расположение в цепи интересующих нас групп тройками, разделенными группами с "большими" нормальными 2-подгруппами, и существование тройки спорадических простых групп $M(22), M(23)$ и $M(24)^{\prime}$ (п. (vi) теоремы Фишера), также интригуют. Возникает вопрос, не является ли такая деталь проявлением некой общей закономерности?

Наконец, полученные результаты позволяют выдвинуть следующую гипотезу о более тесной связи групп $W_{n} \in\left\{S p_{2 l}(2), O_{2 l}^{ \pm}(2)\right\}$ с группами Кокстера $G_{n}$.

$\Gamma$ и п о т е з а. Группа $W_{n}$ - это единственная конечная фактор-группа группы $G_{n}$, обладающая тривиальным центром и простым неабелевым коммутантом.

\section{СПИСОК ЛИТЕРАТУРЫ}

1. Fischer B. Finite groups generated by 3-transpositions // WMI Preprints. Coventry (UK): University of Warwick, 1969.

2. Горенстейн Д. Конечные простые группы. М.: Мир, 1985. 352 p.

3. Hall J.I. Graphs, geometry, 3-transposition, and symplectic $F_{2}$-transvection groups // Proc. London Math. Soc. 1989. Vol. 58. P. 89-111.

4. Созутов А.И. О группах типа $\Sigma_{4}$, порожденных 3-транспозициями // Сиб. мат. журн. 1992. T. 33, № 1. C. 140-149.

5. McLaughlin J. Some subgroups of $S L_{n}\left(F_{2}\right) / /$ Ill. J. Math. 1969. Vol. 13, no. 1. P. 108-115.

6. Aschbacher M. 3-transposition groups. Cambridge: Cambridge University Press, 1997. 260 p.

7. Matsuo A. 3-transposition groups of symplectic type and vertex operator algebras // J. Math. Soc. Japan. 2005. Vol 57, № 3. P. 639-649.

8. Созутов А.И., Кузнецов А.А., Синицин В.М. О системах порождающих некоторых групп с 3-транспозициями // Сиб. мат. электрон. изв. 2013. Т. 10. С. 285-301. doi: 10.17377/semi.2013.10.022 .

9. Созутов А.И. Об алгебрах Ли с мономиальным базисом // Сиб. мат. журн. 1993. Т. 34, № 5. C. $188-201$.

10. Hall J.I., Shpectorov S. The spectra of finite 3-transpositions groups [e-resource]. 2018. 35 p. URL: arXiv:1809.03696.

11. Griess R.L. Jr. A vertex operator algebra related to $E_{8}$ with avtomorphism group $O^{+}(10,2) / /$ Ohio State Univ. Math. Res. Inst. Vol. 7. Berlin: Publ. de Gruyter, 1998. P. 43-58.

12. Cuypers H., Horn M., J. in 't panhuis, Shpectorov S. Lie algebras and 3-transpositions // J. Algebra. 2012. Vol. 368. P. 21-39. doi: 10.1016/j.jalgebra.2012.06.010.

13. Бурбаки Н. Группы и алгебры Ли. Группы, порожденные отражениями. Гл. IV-VI. М.: Мир, 1972. $334 \mathrm{c}$.

14. Коксетер Г.С.М., Мозер У.О.Дж. Порождающие элементы и определяющие элементы дискретных групп. М.: Наука, 1980. 240 с.

15. Кондратьев А.С. Группы и алгебры Ли. Екатеринбург: Изд-во УрО РАН, 2009. 310 с. 
16. О’Мира О. Лекции о симплектических группах. М.: Мир, 1979. 167 с.

17. Conway J.H. , Curtis R.T., Norton S.P., Parker R.A., Wilson R.A. An atlas of finite groups. Oxford: Clarendon Press, 1985. 252 p.

Поступила 19.05.2020

После доработки 4.11.2020

Созутов Анатолий Ильич

Принята к публикации 16.11.2020

д-р физ.-мат. наук, профессор

Сибирский федеральный университет

г. Красноярск

e-mail: sozutov_ai@mail.ru

Синицин Владимир Михайлович

Сибирский федеральный университет

г. Красноярск

e-mail: sinkoro@yandex.ru

\section{REFERENCES}

1. Fischer B. Finite groups generated by 3-transpositions. WMI Preprints, Coventry (UK): University of Warwick, 1969.

2. Gorenstein D. Finite simple groups. An introduction to their classification. University Series in Mathematics, N Y: Plenum Publishing Corp., 1982, 333 p. ISBN: 0-306-40779-5 . Translated to Russian under the title Konechnye prostye gruppy. Vvedenie v ikh klassifikatsiyu. Moscow: Mir Publ., 1985, 352 p.

3. Hall J.I. Graphs, geometry, 3-transposition, and symplectic $F_{2}$-transvection groups. Proc. London Math. Soc., 1989, vol. 58, no. 1, pp. 89-111. doi: 10.1112/plms/s3-58.1.89.

4. Sozutov A.I. Groups of type $\Sigma_{4}$ generated by 3-transpositions. Siberian Math. J., 1992, vol. 33, no. 1, pp. 117-124. doi: 10.1007/BF00972943.

5. McLaughlin J. Some subgroups of $S L_{n}\left(F_{2}\right)$. Ill. J. Math., 1969, vol. 13, no. 1, pp. 108-115. doi: $10.1215 / \mathrm{ijm} / 1256053741$.

6. Aschbacher M. 3-transposition groups. Cambridge: Cambridge University Press, 1997, 260 p. ISBN: 0-521-57196-0

7. Matsuo A. 3-transposition groups of symplectic type and vertex operator algebras. J. Math. Soc. Japan, 2005, vol. 57, no. 3, pp. 639-649. doi: 10.2969/jmsj/1158241926 .

8. Sozutov A.I., Kuznetsov A.A., Sinitsin V.M. Systems of generators of some groups with 3-transpositions. Sib. Elektron. Mat. Izv., 2013, vol. 10, pp. 285-301 (in Russian). doi: 10.17377/semi.2013.10.022 .

9. Sozutov A.I. On Lie algebras with monomial basis. Siberian Math. J., 1993, vol. 34, no. 5, pp. 959-971. doi: $10.1007 / \mathrm{BF} 00971409$.

10. Hall J.I., Shpectorov S. The spectra of finite 3-transpositions groups [e-resource]. 2018. 35 p. Available at: arXiv:1809.03696.

11. Griess R.L., Jr. A vertex operator algebra related to $E_{8}$ with avtomorphism group $O^{+}(10,2)$. In: The Monster and Lie algebras. Ohio State Univ. Math. Res. Inst., vol. 7. Berlin: Publ. de Gruyter, 1998, pp. 43-58. ISBN: 9783110161847.

12. Cuypers H., Horn M., in 't panhuis, Shpectorov S. Lie algebras and 3-transpositions J. Algebra, 2012, vol. 368, pp. 21-39. doi: 10.1016/j.jalgebra.2012.06.010.

13. Bourbaki N. Groupes et algebres de Lie (Chapt. IV-VI). Paris: Hermann, 1968, 282 p. doi: 10.1007/9783-540-34491-9. Translated to Russian under the title Gruppy i algebry Li (glavy IV-VI), Moscow: Mir Publ., 1972, 334 p.

14. Coxeter H.S.M., Moser W.O.J Generators and Relations for Discrete Groups. Berlin; Heidelberg: Springer-Verlag, 1972, 164 p. doi: 10.1007/978-3-662-21946-1. Translated to Russian under the title Porozhdayushchie elementy i opredelyayushchie elementy diskretnykh grupp, Moscow: Nauka Publ., 1980, $240 \mathrm{p}$. 
15. Kondrat'ev A.S. Gruppy i algebry Li [Groups and Lie algebras]. Ekaterinburg: UrO RAN Publ., 2009, 310 p. ISBN: 978-5-7691-2111-1.

16. O'Meara O.T. Symplectic groups. Providence, R.I.: Amer. Math. Soc., 1978, 125 p. ISBN: 0-8218-1516-4 . Translated to Russian under the title Lektsii o simplekticheskikh gruppakh, Moscow: Mir Publ., 1979, $167 \mathrm{p}$.

17. Conway J.H., Curtis R.T., Norton S.P., Parker R.A., Wilson R.A. Atlas of finite groups. Oxford: Clarendon Press, 1985, 252 p. ISBN: 0198531990.

Received May 19, 2020

Revised November 4, 2020

Accepted November 16, 2020

Funding Agency: This work was supported by the Russian Foundation for Basic Research (project no. 19-01-00566 A.)

Sozutov Anatoly Ilich, Dr. Phys.-Math. Sci., Prof., Siberian Federal University, Krasnoyarsk, 660041 Russia, e-mail: sozutov_ai@mail.ru .

Vladimir Mihaylovich Sinitsin, Siberian Federal University, Krasnoyarsk, 660041 Russia, e-mail: sinkoro@yandex.ru .

Cite this article as: V. M. Sinitsin, A. I. Sozutov. On the connection of some groups generated by 3-transpositions with Coxeter groups, Trudy Instituta Matematiki i Mekhaniki UrO RAN, 2020, vol. 26 , no. 4, pp. 234-243. 